built. The $\mathrm{FK}_{3}$ supplementary stars will soon be added to this list. In addition to such well-recognized star lists, many special lists of stars have found their way into our fundamental programmes. Among the special lists are the PZT lists for Washington, Richmond, Herstmonceux and Ottawa and a selection of $\mathrm{O}$ and $\mathrm{B}$ type stars from the list prepared by Dr A. Blaauw. A considerable portion of our observing time since r94I has been devoted to about 7200 reference stars between $+35^{\circ}$ declination and the North Pole.

For some time we have been deeply concerned with the sparsity of stars in the $\mathrm{FK}_{3}$, especially when endeavouring to compare our fundamental catalogues with a standard reference system or with similar catalogues produced elsewhere. To remedy this we have recently resorted to comparing through the GC because of its greater number of stars, a procedure which is not entirely satisfactory. The addition of the supplementary stars to the $\mathrm{FK}_{3}$ will greatly increase its value as a catalogue of comparison.

\title{
6. ON THE FUNDAMENTAL ASTROMETRIC WORK OF THE CAPE OBSERVATORY
}

\author{
By R. H. STOY
}

At the Cape we are engaged on two major astrometric projects. The first of these, the Cape Photographic Catalogue for I950.0, includes 65,000 stars from $-30^{\circ}$ to $-40^{\circ}$ and from $-52^{\circ}$ to the South Pole. It is a differential rather than a fundamental catalogue, but it should provide the material from which a really satisfactory selection of faint stars for a fundamental catalogue may be made. The astrometric observations are complete and it is hoped that the work as a whole will be finished by 1960 .

The second astrometric project is the series of fundamental observations with the Gill Reversible Transit Circle which is to form the basis of the Second Cape Catalogue for I950.0. The working list now contains approximately 7000 stars and includes the usual Cape clock and azimuth stars, all $\mathrm{FK}_{3}$ stars south of $-20^{\circ}$, all GC stars brighter than $8^{\mathrm{m}} \cdot 5$ between $-30^{\circ}$ and $-52^{\circ}$ and between $-80^{\circ}$ and the South Pole, a selection of stars south of $-80^{\circ}$ to serve as standards for the reduction of the photographic zone observations, and a number of extra stars added recently. These extra stars include 396 stars between $-29^{\circ} 30^{\prime}$ and $-40^{\circ}$ which, together with the stars already in the working list, are intended to provide a sufficient number of evenly distributed stars for the reduction of photographic plates to be taken with the Yale Observatory Camera in Australia. They also include 65 stars south of $-30^{\circ}$ taken from FKSZ list of 93I faint fundamental stars. The other 22I stars of this list south of $-30^{\circ}$ were already included in the working list or have been observed with the Gill Reversible Transit Circle within the last ten years. It has not been considered necessary to add to the present working list any of the 845 stars from the recently published $\mathrm{FK}_{3}$ Supplementary List, as all these stars were either already included or have been observed at the Cape relatively recently. Our intention is to observe, as far as it may be possible, each star eight times, four times in each position of the instrument. Observations of the Sun, Mercury, Venus and a number of bright stars are attempted five days a week. The Moon, Ceres, Juno, Pallas and Vesta are observed whenever possible and other planets twice a week. The observations for this catalogue were begun at the end of I95I and it is hoped that they will be completed before the end of I957. Those for the polar section are already completed and are to be reduced together with the corresponding photographic observations before the remainder of the catalogue.

The observations made with the Gill Reversible Transit Circle have entered so fully into the constitution of the southern portions of the various fundamental catalogues that it may be thought that future work with it can do little to improve the present fundamental systems. This rather pessimistic view may be partially true and undoubtedly some of the present systematic errors can be discovered and eliminated more easily by 
series of observations with rather more specialized instruments, such as those undertaken by Drs Schmeidler and van Herk. On the other hand, there are various points about the Cape work that still require further investigation and that may lead to small systematic corrections to the observed fundamental system. In so far as the right ascensions are concerned, the rather large pivot errors, and particularly their change with time, need to be closely studied. Several possible systematic errors which may effect observations of declination have recently been pointed out (e.g. possible change of flexure with temperature (x), change of refraction with spectral type(2), change of refraction with humidity (3)), and these possibilities ought to be carefully examined under the conditions obtaining at the Cape.

To some extent the accuracy of the observed fundamental system may be checked either by altering the method of observing or by making changes to the instrument. The method of observing right ascensions was slightly changed in I944 and since I95I the declination circle reading has been recorded photographically. The movable circle is being regraduated by Messrs Hilger and Watts, but will not be brought into use before the beginning of the next catalogue. By that time we hope to have improved the method by which the declination circle is photographed, to have had the pivots reground, to have improved the method by which the bright stars are dimmed and to have improved the collimators.

The composition of the next working list for the transit circle is now under consideration. Two interlinked programmes seem to be equally urgent. The first is the observation of the various groups of standard stars in as fundamental a way as possible. The second is the extension of the resulting fundamental system to a sufficient number of stars to ensure the adequate reduction of photographic observations. As only one transit circle is available, we propose to observe both programmes concurrently. Certain nights of the week will be devoted entirely to observations of stars in the fundamental list and the remaining nights will be used for observing stars in the secondary list and a small selection of the fundamental stars. The secondary stars will be observed fundamentally but reduced differentially. The observed 'night corrections' will then be available for strengthening the system of the fundamental stars. The fundamental stars must be observed simultaneously all over the sky; the secondary stars can be observed in zones. The observations of the fundamental stars will be spread over several years, but those for any zone of secondary stars will be concentrated in as short a time as possible.

The choice of the stars for the fundamental list is fairly clear, viz. all stars south of the equator in the FK 3 , the FK 3 Supplementary List and the FKSZ, together with a number of stars selected from these lists between the equator and $+30^{\circ}$. The choice for the secondary stars is not so clear. In the zones north of $-30^{\circ}$ much will depend on what collaboration can be arranged with observatories in the northern hemisphere. South of $-30^{\circ}$, the choice is still open. The questions that have to be decided are: 'What kind of stars and how many of them? Will it be better to get several meridian observations each for relatively few stars or a few meridian observations each for relatively many stars?' As the weight of the transfer of the system of the fundamental stars to the secondary stars will be directly proportional to the number of observations made, the alternative schemes appear to have equal weight. We are therefore inclined to favour the second, since the accuracy of the individual star places can be increased by photographic observations with our astrometric camera.

Though much of the photographic work in the future may be done with lenses covering fields of $10^{\circ} \times 10^{\circ}$ or more, it is probably wiser to make provision for lenses covering smaller fields than this. We are therefore contemplating a density for these secondary stars of approximately one star per square degree. Thus there will be about ro,ooo stars south of $-30^{\circ}$ and we intend observing each of these stars four times with the transit circle and measuring its place on at least four photographic plates. These plates will be so arranged that every star will appear on a different quadrant on each of four plates. The resulting star places would be still further strengthened if some other observatory were to observe the same zones simultaneously with a different camera. To enable each 
zone to be covered in approximately two years, the sky south of $-30^{\circ}$ will be divided into four zones and we propose to observe them in the order $-40^{\circ}$ to $-52^{\circ},-30^{\circ}$ to $-40^{\circ},-52^{\circ}$ to $-64^{\circ}$ and $-64^{\circ}$ to the South Pole.

The ro,000 stars must be chosen to ensure as much homogeneity as possible with the catalogues contemplated north of $-30^{\circ}$. 5000 should satisfy the criteria laid down by Zverev for the KSZ. The other 5000 can be selected from approximately the same range of visual magnitude, but with a greater variety of spectral type. Many of these stars can be chosen from those already used as standards for the Cape Photographic Catalogue for I950.0. For the zones south of $-35^{\circ}$, these stars were chosen so as to be evenly distributed, but a critical examination of them will be necessary to see how far it is possible to improve the distribution as regards magnitude and colour.

To test the practicability of the proposed criteria, Mr Lourens has made an experimental selection of stars between $-30^{\circ}$ and $-64^{\circ}$ for two areas, the one near the galactic pole (where the choice is most difficult) and the other near the galactic equator. The following statistics for this preliminary selection which covers 464 square degrees and includes 243 stars satisfying Zverev's criteria ( $Z$ stars) and 232 additional stars (A stars) may be of some interest.

Distribution in $m_{p v}$ :

\begin{tabular}{|c|c|c|c|c|c|c|}
\hline$m_{p v}$ & $<7 \cdot 4$ & $7 \cdot 5-7 \cdot 9$ & $8 \cdot 0-8 \cdot 4$ & $8 \cdot 5-8 \cdot 9$ & $9 \cdot 0-9 \cdot 4$ & $>9 \cdot 4$ \\
\hline Percentage $Z$ stars & $1 \cdot 2$ & 9.9 & $22 \cdot 2$ & $\mathbf{3 3} \cdot \mathbf{8}$ & $30 \cdot 5$ & $2 \cdot 5$ \\
\hline Percentage A stars & $3 \cdot 0$ & $13 \cdot 0$ & $21 \cdot 6$ & $26 \cdot 4$ & $31 \cdot 2$ & $4 \cdot 8$ \\
\hline Percentage all stars & $2 \cdot 1$ & $11 \cdot 4$ & $21 \cdot 9$ & $30 \cdot 2$ & $30 \cdot 8$ & $3 \cdot 6$ \\
\hline
\end{tabular}

Brightest star: $7 \cdot 0(Z), 6 \cdot 9$ (A).

Faintest star: $9 \cdot 7(\mathrm{Z}), 9 \cdot 8(\mathrm{~A})$.

Mean magnitude, high latitude: $8 \cdot 68(\mathrm{Z}), 8 \cdot 70$ (A) ;

low latitude: $\mathbf{8 \cdot 5 4}(\mathrm{Z}), \mathbf{8 \cdot 4 0}(\mathrm{A})$.

Distribution in colour:

$\begin{array}{lcccccc}\quad \text { Sp type } & B & A & F & G & K & M \\ \text { Percentage Z stars } & 0 \cdot 0 & \mathbf{2 \cdot 1} & \mathbf{1 4 \cdot 4} & \mathbf{1 8 \cdot 1} & \mathbf{6 3 \cdot 0} & \mathbf{2 \cdot 5} \\ \text { Percentage A stars } & \mathbf{0 . 9} & \mathbf{9 . 5} & \mathbf{1 5 \cdot 6} & \mathbf{2 9 \cdot 0} & \mathbf{4 2 \cdot 0} & \mathbf{3} \cdot 0 \\ \text { Percentage all stars } & \mathbf{0 . 4} & \mathbf{5 \cdot 7} & \mathbf{1 5 \cdot 0} & \mathbf{2 3 \cdot 4} & \mathbf{5 2 \cdot 8} & \mathbf{2 \cdot 7}\end{array}$

Mean colour index, high latitude: $+0.85(\mathrm{Z}),+0.67(\mathrm{~A})$;

low latitude: $+1.04(\mathrm{Z}),+0.76(\mathrm{~A})$.

$89 \%$ of the Zverev stars and $60 \%$ of the additional stars have P.M.'s less than 0.040 per year. The mean P.M.'s for the high-latitude areas are 0.027 per year for the Zverev stars and $0 " 048$ per year for the additional stars. The corresponding figures for the low latitudes are 0.026 and $0 " 036$.

In comparing these statistics with similar ones for selections of northern stars, the differences in the magnitude scales used must be remembered. For these southern stars, the magnitudes and colours used are on the $1953 \mathrm{~S}$ system which has a normal scale. Thus faint stars appear to be relatively fainter than they would, had their magnitudes been expressed on many of the scales current in ordinary meridian catalogues. The approximate working limit on this scale for the Gill Reversible Transit Circle is $9.5 S P v$ at the altitude of the South Pole and a little fainter at the zenith.

Prof. Heckmann and Prof. Brouwer expressed a great debt of gratitude to the Cape Observatory and to its director for this bold undertaking which will be of major importance for astronomy.

Prof. Brouwer referred to the new photographic zone observations south of $-30^{\circ}$, to be undertaken by the Yale Observatory in co-operation with the Sydney Observatory (4). In addition, he intends to take a series of experimental plates near the south celestial pole. Four plates are to be centred on the pole, to be taken $6 \mathrm{hr}$. apart in sidereal 
time; r6 centred on $-85^{\circ}, 20$ on $-80^{\circ}, 24$ on $-75^{\circ}$. Each plate covers an area $10^{\circ} \times 10^{\circ}$. The purpose of this experiment is to examine whether, with this degree of overlapping, it is possible to $(a)$ increase the systematic accuracy of the reference-star system, $(b)$ use fewer stars than have been considered necessary heretofore. He referred to suggestions along these lines made by Dr Stoy(s) at the Evanston Conference.

Dr Dieckvoss stressed the importance of using overlapping plates (cf. the Paris Catalogue of $I I, 755$ stars), and also of measuring plates in strictly orthogonal system in order to strengthen the value of photographic positions.

\title{
REFERENCES
}

(I) F. Schmeidler, Vistas in Astronomy, pt. XI.

(2) G. van Herk, B.A.N. 9, 489, I952.

(3) F. P. Scott, $A . J .60,93$, 1955.

(4) See this volume, p. II 7 .

(5) R. H. Stoy, A.J. 59, 39, 1954.

\section{REPORT ON OBSERVATIONS OF SOUTHERN FUNDAMENTAL DECLINATIONS IN PROGRESS AT MT STROMLO OBSERVATORY}

\author{
BY F. SCHMEIDLER
}

The idea of the observations of southern fundamental declinations at Mt Stromlo follows a suggestion first made by Dneprovski ( $A . N$. 24I, 2I9) that the same stars should be observed by the same observer with the same instrument at two stations, one in each hemisphere. Dneprovski's idea was to find out whether the extreme $\Delta \delta_{\delta}$-errors, resulting at that time from most vertical circles and also some meridian circles, were either purely instrumental errors, or real errors in fundamental declinations.

Although it was shown in I933 by Rabe $(A . N . \mathbf{2 4 8}, 369)$ that in most cases the extreme $\Delta \delta_{\delta}$-errors could be removed by applying the right amount of flexure corrections to the respective observations, some unexplained differences remained which were of a smaller amount. According to Kopff $(A . N . \mathbf{2 6 9}, \mathbf{x 6 0})$ the differences between the declinations of $\mathrm{FK}_{3}$ and GC are as large as $0 " 4$ in some parts of the sky, especially at high southern declinations. Therefore it seemed still worth while carrying out Dneprovski's original idea.

The importance of observations of fundamental southern declinations is also supported in view of the revision of $\mathrm{FK}_{3}$, as pointed out recently by Kopff $(M . N$. Ir4, 478). There are only a very small number of catalogues of southern fundamental positions, even fewer than there were when the $\mathrm{FK}_{3}$ itself was compiled. Furthermore, Cape and perhaps Cordoba are the only observatories where, at present, regular meridian observations of southern stars are made, so that any systematic errors, by which the observations of one of these two observatories may be affected, enter with very great weight into the final fundamental system.

The author has been observing fundamental declinations with the Munich vertical circle since I940; his programme of observation of all Auwers stars north of $-30^{\circ}$ declination was completed in I953. The very generous assistance of the Deutsche Forschungsgemeinschaft as well as of the Commonwealth Observatory at Mt Stromlo enabled him to go to Australia in February I954 to make observations of southern fundamental declinations. The actual observations began in May I954 and will probably be finished by September I955.

The proposed programme includes all Auwers stars south of declination $+30^{\circ}$ and also all Zusatzsterne south of the equator. Therefore all Auwers stars in the belt between declinations $-30^{\circ}$ and $+30^{\circ}$ will be observed from both hemispheres and it is hoped to 